\title{
Immunocontraception of African elephants
}

\author{
A humane method to control elephant populations without behavioural side effects.
}

$\mathrm{C}$ oncerted efforts to nurture elephant populations have resulted in elephant overpopulation in several areas, which in turn has led to damaging levels of browsing. As an alternative to culling entire family groups in order to control this damage, we have developed an immunocontraceptive vaccine from pig zona pellucida which safely and successfully controls freeroaming African elephants.

Immunocontraceptive vaccines cause the immune system to produce antibodies that prevent fertilization, without the side effects of hormonal contraceptives. The vaccine antigens are the proteins of the zona pellucida, the clear protein coat surrounding mammalian eggs. The surface structures of the elephant zona pellucida are very similar to those of the pig zona pellucida $(\mathrm{pZP})^{1-3}$.

Female zoo elephants vaccinated with pZP and an adjuvant all developed antibodies that persisted for $12-14$ months ${ }^{1,2}$, at a level equivalent to those found in horses given immunocontraception ${ }^{4,5}$. Based on this, we planned field trials in Kruger National Park, in conjunction with the South African National Parks.

Initial trials using 41 adult female elephants tested the efficacy of pZP as an immunocontraceptive. Elephants were located from a helicopter, and females to be anaesthetized (by aerial darting) were identified as non-pregnant by the presence of a calf smaller than 1 metre high. We then used ultrasound scans to confirm that females were not pregnant, and all nonpregnant animals were bled to obtain prevaccination serum samples. Twenty-one elephants were given an initial vaccination of pZP with adjuvant; 20 controls received a placebo.

All treated elephants were fitted with radiocollars. The control females were fitted with numbered collars and paired in a family unit with a vaccinated elephant. The vaccinated elephants were located 6 weeks later and received a first booster, followed by another 6 months later. Both boosters were administered remotely with drop-out darts from a helicopter.

Twelve months after the initial vaccination, the elephants were recaptured and scanned for pregnancy. Of those treated with pZP, 19 were recaptured (two were not found because their radiocollars failed). Nine of the 19 were pregnant, ten were not. One of the pregnant elephants was in the last trimester of gestation (22 months) and gave birth to a healthy calf, showing that the vaccination of a pregnant elephant with
pZP has no effect on gestation, the fetus or parturition. Eighteen of the 20 control elephants were located, 16 of which were pregnant. Therefore, significantly $\left(\chi^{2}, P=0.005\right)$ fewer vaccinated elephants $(44 \%, 8 / 18)$ were pregnant than the control females (89\%, 16/18).

Subsequently, we vaccinated ten elephants using a revised schedule. Females received an initial vaccination, followed by identical boosters delivered from a helicopter two and four weeks later. All elephants were fitted with radiocollars; five had global positioning satellite (GPS) collars (Lotek, Newmarket, Canada) which recorded their location hourly.

Of the ten elephants, two (20\%) were pregnant after 10 months. This was significantly $\left(\chi^{2}, P=0.001\right)$ lower than the conception rate of the control elephants $(89 \%$, $16 / 18)$ and initial immunocontraception rates $(44 \%, 8 / 18)$.

Female elephants with oestradiol implants have shown aberrant behaviour by separating off within the family unit (D.G., personal observation). GPS-collar location data indicated that there was no abnormal separation of the vaccinated females within a family unit over 8 months. This, combined with field observations of vaccinated females, suggests that the immunocontraceptive vaccine causes no behavioural abnormalities.

Finally, we tested the reversibility of immunocontraception, and its application for a second consecutive year. Of seven elephants from the group that had initially received immunocontraception, four were vaccinated with $\mathrm{pZP}$ and adjuvant, and

\section{Evolutionary biology}

\section{Sexual conflict and speciation}

exual conflict occurs because males are selected to produce as many offspring as possible, even if this means lowering the overall reproductive output of individual females. A new model proposed by Gavrilets ${ }^{1}$ suggests that strong asymmetries between males and females in the costs and benefits of mating will create runaway coevolution between the sexes, promoting rapid divergence between populations and hence speciation. This is an intriguing possibility, not least because it runs counter to existing models ${ }^{2}$ which suggest that greater sexual conflict will result in males mating three were not. Twelve months later, the seven elephants were captured and re-evaluated. Ultrasound scans showed that all three untreated females had conceived again, compared with none of the vaccinated elephants, although all were cycling. This indicates that the vaccine is reversible, and that it has no deleterious effect on the ovary and its cyclicity.

Elephants are intelligent and empathetic mammals, and culling is a last resort in controlling their numbers. Our immunocontraceptive study shows that free-roaming African elephants vaccinated with pZP are protected against conception. This $\mathrm{pZP}$ immunocontraception is safe and reversible and is thus a practical tool for controlling elephant populations.

R. A. Fayrer-Hosken ${ }^{\star}$, D. Grobler $\dagger$,

J. J. Van Altena $\dagger$, H. J. Bertschinger

\section{J. F. Kirkpatrick $\$$}

${ }^{*}$ College of Veterinary Medicine, University of

Georgia, Athens, Georgia 30602-7385, USA

e-mail: rfh@calc.vet.uga.edu

$\dagger$ Kruger National Park, Private Bag X402,

1350 Skukuza, South Africa

$\ddagger$ Department of Theriogenology, University of Pretoria, Private Bag X04, 0110 Onderstepoort, South Africa

\$ZooMontana, 2100 South Shiloh Road, Billings, Montana 59106, USA

1. Fayrer-Hosken, R. A. et al. Theriogenology 47, 397 (1997).

2. Fayrer-Hosken, R. A. et al. Theriogenology 52, 835-846 (1999).

3. Fayrer-Hosken, R. A., Bertschinger, H. J., Kirkpatrick, J. F., Turner, J. W. \& Liu, I. K. M. Bulletin 25, 18-21 (1997).

4. Willis, L. P., Heusner, G. L., Warren, R. J., Kessler, D. \& FayrerHosken, R. A. J. Eq. Vet. Sci. 14, 364-370 (1994).

5. Kirkpatrick, J. F., Turner, J. W. Jr, Liu, I. K., Fayrer-Hosken, R. \& Rutberg, A. T. Reprod. Fertil. Dev. 9, 105-110 (1997).

more indiscriminately, breaking down reproductive barriers between divergent populations. One reason for this difference is that the new model is based on the idea that females can avoid costs of mating if they are incompatible with some males, whereas we suggest that in reality this may rarely be the case.

Gavrilets' model ${ }^{1}$ assumes a quadratic relation between female fitness and the proportion of the male population with which she is compatible (morphologically, physiologically or genetically). The shape of this relationship is not theoretically derived (as it might be), but is the simplest function under which there is an intermediate optimum proportion of compatible males. This relationship is based on observations suggesting that females experiencing unusually 
high or low mating rates may have reduced fitness ${ }^{3}$, but such analyses must be treated with caution. Females are expected to be adapted to the number of matings they experience ${ }^{4}$, even if this is a compromise between their optimal mating rate and that which males attempt to impose.

More significantly, most of the costs to females that Gavrilets sees as driving sexual conflict (such as predation, sensory exploitation, different costs of mating or seminal fluid toxicity $)^{1}$ are paid as a result of matings per se, and hence can only be prevented if matings themselves are avoided. However, if incompatibilities prevent mating, then males are expected to be able to determine rapidly whether a particular female is compatible. Hence incompatibilities that prevent mating between particular male and female phenotypes will simply result in males only attempting to mate with females with whom they are compatible, with no reduction in the number of matings or attempted matings to which each female is subjected.

This suggests that the potential for sexual conflict to promote divergence may be limited to species without the possibility of pre-copulatory mate choice, but in which there are still costs to being compatible with too many males. There may be examples of such species - for instance, broadcast spawners may suffer from polyspermy but such cases are likely to be rare.

Although we question the generality of Gavrilets' model ${ }^{1}$, it does indicate the potential for the different priorities of males and females to drive evolution of reproductive isolation. Perhaps rather than lumping so much biology together under the title 'sexual conflict', we need to consider the specific processes involved.

Tom Tregenza, Roger K. Butlin, Nina Wedell School of Biology, University of Leeds,

Leeds LS2 9JT, UK

e-mail: gentbt@leeds.ac.uk

1. Gavrilets, S. Nature 403, 886-889 (2000).

2. Parker, G. A. \& Partridge, L. Phil. Trans. R. Soc. Lond. B 353 , 261-274 (1998).

. Arnqvist, G. \& Nilson, T. Anim. Behav. (in the press).

4. Holland, B. \& Rice, W. R. Proc. Natl Acad. Sci. USA 96,

5083-5088 (1999).

Gavrilets replies - Tregenza et al. maintain that my model's predictions ${ }^{1}$ run counter to the model of Parker and Partridge ${ }^{2}$, but it is not straightforward to compare these two classes of model because of their inherent differences.

First, my model considers genetic divergence of allopatric populations, whereas the models developed in ref. 2 describe the evolutionary consequences of a secondary contact between populations that have already diverged: that is, they model reinforcement of reproductive isolation rather than its emergence. Second, Parker and Partridge ${ }^{2}$ use an evolutionarily stable strategy framework with a set of fixed evolutionary options, whereas I model continuous coevolution of the sexes, during which evolutionary options are continuously changing.

In spite of these differences and contrary to the claim of Tregenza et al., there is no contradiction in the predictions of both types of model. Parker and Partridge propose that mating conflict could be either a hindrance to isolation if 'male-win' scenarios prevail, or a facilitator if females tend to win - exactly as predicted by my model if coevolution is restricted. However, my model takes an additional step by considering the possibility of continuous coevolution of the sexes. In this case the prediction is that neither sex will win the sexual conflict, but rather that there will be a dynamic coevolutionary compromise.

Tregenza et al. question the generality of my model's assumption that females have an intermediate optimum mating rate, as well as the specific (quadratic) function I used to model the relation between a female's fitness and her mating rate: in fact, the model is well supported by insect ${ }^{3}$ and other data ${ }^{4,5}$, and the idea that excessive mating rates are bad for females is the essence of sexual conflict.

Inadequate mating rates are also detrimental ${ }^{6}$. Thus, with sexual conflict the optimum mating rate must be intermediate. As for the shape of the relation between female mating costs and mating rate, more data are indeed necessary. However, I do not anticipate that using functions more complicated than a quadratic one will affect my main conclusions ${ }^{1}$.

Tregenza et al. suggest that, in most species, males will rapidly identify and attempt to mate only with those females with whom they are compatible, thus reducing the potential for sexual conflict to promote divergence. But even if the necessary 'indicators' of female compatibility should be readily available and the males smart enough to exploit them, in a polymorphic population different females will experience a variable number of matings. This will induce fitness differences and initiate runaway coevolution, resulting in genetic divergence of isolated populations. Although the model does not describe all the specific processes involved in sexual conflict, other weaknesses are not yet apparent.

\section{S. Gavrilets}

Department of Ecology and Evolutionary Biology,

Department of Mathematics, University of

Tennessee Knoxville, Tennessee 37996, USA

1. Gavrilets, S. Nature 403, 886-889 (2000).

2. Parker, G. A. \& Partridge, L. Phil. Trans. R. Soc. Lond. B 353, 261-274 (1998).

3. Arnqvist, G. \& Nilson, T. Anim. Behav. (in the press).

4. Fowler, K. \& Partridge, L. Nature 338, 760-761 (1989).

5. Gems, D. \& Riddle, D. L. Nature 379, 723-725 (1996).

6. Jennions, M. D. \& Petrie, M. Biol. Rev. 75, 21-64 (2000).

\section{Sandwich films \\ Properties of a new soft magnetic material}

he development of advanced electromagnetic devices has been constrained

by a lack of soft magnetic materials with a suitably high saturation magnetization (over 20 kilogauss) and a large permeability roll-off frequency (greater than 1 gigaherz). For example, magnetic hard-disk-drive technology is rapidly approaching the perceived superparamagnetic limit at which the stored bits become thermally unstable ${ }^{1}$ — disks with higher anisotropy are more stable but are not usable because magnetic write heads become saturated. Here we describe a new soft magnetic material with a saturation magnetization of 24 kilogauss and a large permeability of 1,000-1,400 in a wide frequency range of up to about 1.2 gigaherz. This new material promises to have wide application in devices such as magnetic recording heads and integrated inductors.

Soft magnetic materials such as $\mathrm{Co}_{0.57} \mathrm{Ni}_{0.13} \mathrm{Fe}_{0.30}$ (ref. 2) and FeAlN (ref. 3) have a saturation magnetization of about 20 $\mathrm{kG}$, and $\mathrm{Fe}_{1-x} \mathrm{Co}_{x}$ alloys (where $0.3 \leqslant x \leqslant 0.4$ ) have the highest saturation magnetization that occurs in nature (about $24.5 \mathrm{kG}$ ). However, the coercivity of these FeCo alloys tends to be greater than 5 oersteds, making them unsuitable for magnetic write heads 4 . Attempts to obtain soft magnetic materials have involved making artificial structures ${ }^{5}$.

We have created a sandwich structure, $\mathrm{Ni}_{0.81} \mathrm{Fe}_{0.19} /\left(\mathrm{Fe}_{0.7} \mathrm{Co}_{0.3}\right){ }_{0.95} \mathrm{~N}_{0.05} / \mathrm{Ni}_{0.81} \mathrm{Fe}_{0.19}$, in which the FeCoN film is $100 \mathrm{~nm}$ thick, and each $\mathrm{Ni}_{0.81} \mathrm{Fe}_{0.19}$ Permalloy layer is $5 \mathrm{~nm}$ thick, comprising only $4.5 \%$ of the volume of the sandwich. Typical hysteresis loops (magnetization, $M$, plotted against magnetic field, $H$ ) of the sandwich films, determined by using a vibrating sample magnetometer, are shown in Fig. 1, inset:

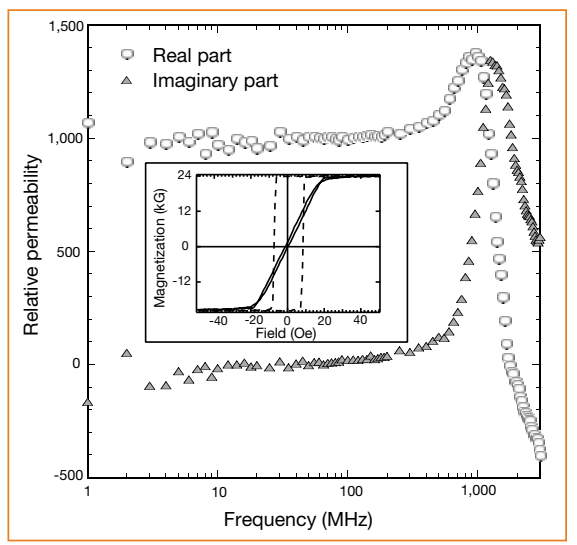

Figure 1 Relative permeability, both real and imaginary parts, versus frequency for a typical $\mathrm{Ni}_{0.81} \mathrm{Fe}_{0.19}(5 \mathrm{~nm}) /\left(\mathrm{Fe}_{0.7} \mathrm{Co}_{0.3}\right)_{0.95} \mathrm{~N}_{0.05}(100 \mathrm{~nm}) /$ $\mathrm{Ni}_{0.81} \mathrm{Fe}_{0.19}(5 \mathrm{~nm})$ film. Inset, typical $M-H$ loops of the sandwich film. Dashed line, easy axis loop; solid line, hard axis loop. 